\title{
Active and Passive Control auto-switching research based on heartbeat protocol for long-distance natural gas pipeline SCADA system
}

\author{
Ye PENG ${ }^{1, a}$ \\ ${ }^{1}$ Trans-Asia Gas Pipeline Co. Ltd., Beijing China \\ apengye_zy@petrochina.com.cn
}

Keywords: heartbeat protocol; SCADA system; long-distance gas pipeline

Abstract: In this paper, long-distance natural gas pipeline system and its SCADA system are generally introduced first. Accordingly, SCADA system control for long-distance gas pipeline is discussed in normal operation. Based on this, control model is established, and needs of auto-switching SCADA's control are discussed. And then, it is proposed that Active and Passive ways of SCADA Control authority auto-switching method based on heartbeat protocol in detail. These make SCADA system self-tested for its telecommunication channel, and robust for the whole SCADA system be greatly improved, and ensure safe operation. At last, some notices for the implementation in different projects are suggested at the end of this paper.

\section{Introduction}

Industrial processes as well as many modern systems depend on SCADA and DCS systems in order to perform their complex functionality [1]. Modern SCADA systems have many sophisticated features, such as distributed architecture, distributed database, GUI interfaces, intelligent RTUs, etc. that are the results of advances in many technologies [2]. These features make control become easier and safer. Also for long-distance natural gas pipelines, SCADA is one of the most important parts of the whole project. With the development of electronic technology and need of saving labor cost, control logic becomes more and more complex, and the whole system can achieve a fully auto-run status.

As known, gas pipeline has its own characteristics, pipeline in a wide geographical area, some Compressor Stations (CS), some Block Valve Stations (BVS), one or two Metering Stations (MS) and one Dispatch Control Center (DCC). For fully-automated operation of the whole system, a model of automatic control shall be precisely considered. And its implementation shall also be well planned.

\section{Control model for gas pipeline}

Usually, there are 3 levels control for daily operation, Central Control, Station Control and Local Control.

a. Central Control

In DCC, information for the whole system is gathered, including hydraulic system analysis, parameters of all the CSs/BVSs/MSs and upstream/downstream status. DCC is also responsible for coordinating with the source and downstream user.

b. Station Control

Station Control is the second operating level. It is responsible for a single station's daily operation. The operator shall control related equipment in Station Control room to make sure of this single station in a normal operation status, and execute orders from DCC to cooperate with upstream and downstream CSs.

\section{c. Local Control}

It is a control from on-site equipment. It only controls single equipment.

In order to make safe operation, a principle shall be ensured: only one instruction shall be executed at one time from different control level, others shall be inhibited.

Based on the above analysis and principle, in order to realize fully automated operation, Central Control shall be guaranteed the highest control authority. When Central Control is offline, to keep 
production continuity, Station Control shall take place in CSs. Local Control level will not be discussed in this paper, due to the single equipment is not enough to keep daily production, and key control parts have been hardwired to Station Control, which is much more reliable than between DCC and CSs.

\section{Active and Passive SCADA control authority auto-switching method Based on heartbeat protocol}

The reliability of SCADA system is mainly affected by two factors: communication security and device failure [3]. Based on the above model, in normal operation, the control authority is on DCC. When the telecommunication is interrupted (such as SDH system being out of work, fiber optical system being cut, satellite system being interrupted), Control Center will not be able to receive real-time data, and carry out effective control on site, which may be hundreds miles away from DCC. At the same time, Station Control System is still waiting for DCC's instruction, which means there is not any control level at that time. The reliability of SCADA system will be greatly influenced. It may easily cause production accident. Hereby, the heartbeat protocol is built in the system for telecom channel status detection. It detects channel's availability, and if the dispatch center are online. According to the result, the system shall distribute the control authority automatically.

Heartbeat mechanism is the most effective technology to achieve the reliable communication of the High Availability (HA) system [4]. The heartbeat protocol is, one side sending signals according to a certain time interval, the other side receiving those signals. According to the signals receiving status, a judgement shall be voted. Heartbeat protocols are used by distributed programs to ensure that if a process in a program terminates or fails, then the remaining processes in the program terminate [5]. For long-distance gas pipeline SCADA system, it can be modelled precisely as a primary and many secondary distributed systems. Hereby, there are two ways of implementation as the following.

(1) Active detection

In accordance with the principles of the heartbeat protocol abovementioned, SCADA system in DCC send signals to SCADA system in CSs at the time interval TA. SCADA system in CSs receives signals, and sets a timer at another time interval TB, and sets a counter at local system. The local counter self-counts at TB (self-add 1 per TB). When CS receives signals from DCC, the counter is reset to 0. If CS cannot receive signals for a certain time, the counter value will reach a pre-set threshold (HA). The telecom-channel between CS and DCC is considered as interruption. Then SCADA system in CS takes control authority, and inhibits all instructions from DCC SCADA system. A message box is popped up on HMI of CS to show this situation to the operator in CS. When problem of this channel is fixed, DCC gets control authority back from CS through server settings. This method is described also as in Fig 1.

(2) Passive detection

Similar to the Active method, the Passive method is SCADA system in CS sending message to DCC at a time interval TC, requesting feedback from DCC. When DCC receives the request, DCC sends feedback signals to CS. Also SCADA system in CS sets a counter locally. The local counter self-counts at TD (self-add 1 per TD). When CS receives feedback from DCC, the counter is reset to 0 . If CS cannot receive feedback for a certain time, the counter value will reach a pre-set threshold (HB). The telecom-channel between CS and DCC is considered with interruption. Then SCADA system control authority is transferred to CS, and inhibits all instructions from DCC SCADA system. And a message box is popped up to show this situation to the operator in CS. Until problem of this channel is fixed, DCC gets control authority back from CS through sever settings. This method is described also as in Fig 2. 


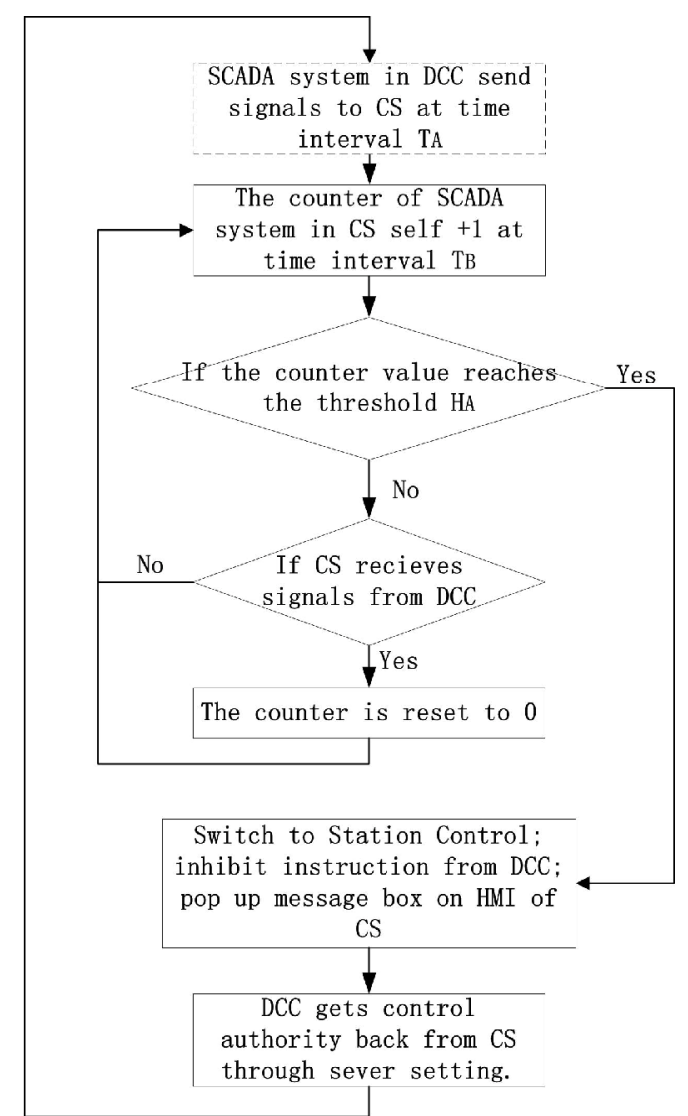

Fig 1 Active detection

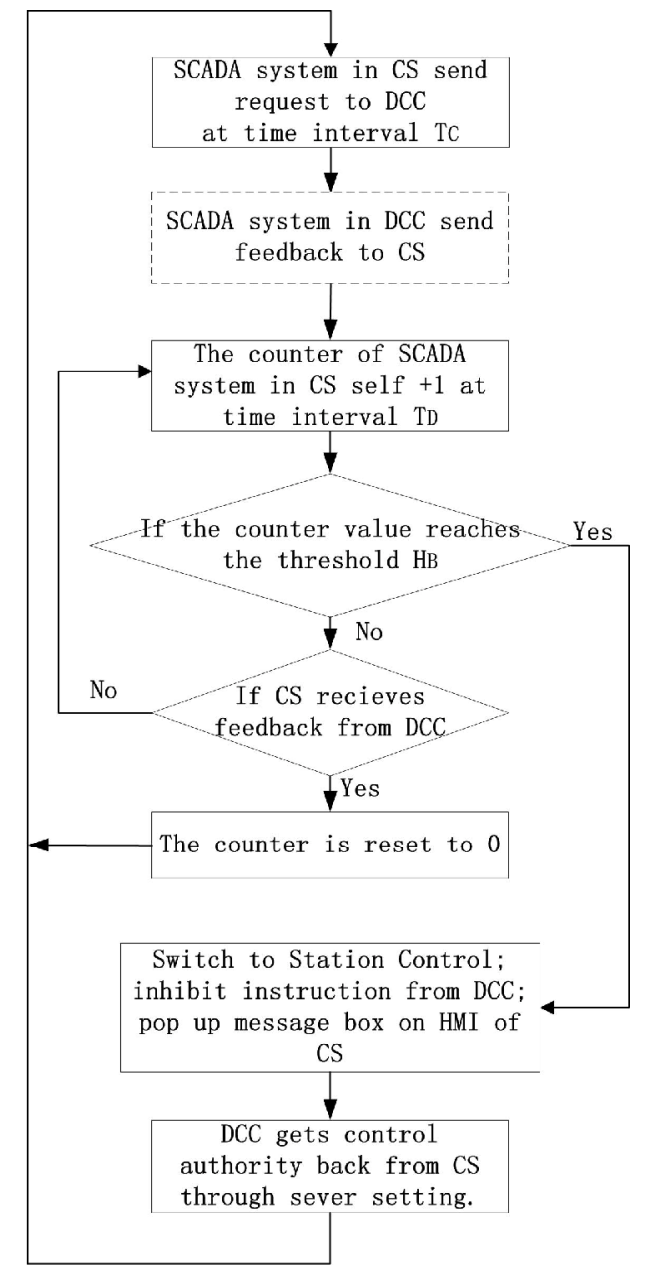

Fig 2 Passive detection 
In Fig 1 and Fig 2, dash line box means SCADA system's action in DCC, whose existence or not will not stop CS's logic entering the next step. In both methods abovementioned, the Active detection way is more simple and intuitive, and implementation is easier, also the system occupies less hardware and software resources. The Passive detection way is more complicated, and the system occupies more hardware and software resources to make more detection with both download and upload channels.

\section{Conclusion}

In this paper, the result of this study is two ways of control auto-switching for SCADA system in long-distance natural gas pipeline, which is based on heartbeat protocol. It makes SCADA system with self-channel-detection function, reduces SCADA system dependence on telecom equipment channel diagnosis function, increases SCADA system's robust and reliability, ensures the safe operation for long-distance gas pipeline, and makes exploration on the future SCADA system and telecom system combination.

It is noteworthy that, to implement this feature, proper configuration shall be set, and relative software functions shall be used for PLCs. Because different manufacture may fabricate different hardware and software functions, to realize the abovementioned methods efficiently, proper architecture shall be carefully considered.

\section{References}

[1] Stamatis Karnouskos, Armando Walter Colombo. Architecting the next generation of service-based SCADA/DCS system of systems. IECON 2011 - 37th Annual Conference on IEEE Industrial Electronics Society. 2011. Page(s): 359 - 364.

[2] Duong Trung. Modern SCADA systems for oil pipelines. Petroleum and Chemical Industry Conference, 1995. Record of Conference Papers., Industry Applications Society 42nd Annua. 1995. Page(s):299 - 305.

[3] Hu Guozhen, Cai tao, Changsong, Duan Shanxu. Solutions for SCADA system Communication Reliability in Photovoltaic Power Plants. IPEMC2009. Page(s): 2482 - 2485.

[4] Gouda, M.G, McGuire, T.M. Accelerated heartbeat protocols. Distributed Computing Systems, 1998. Proceedings. 18th International Conference on. 1998. Page(s): 202 - 209.

[5] Zhanjie Wang, Xiao Li. A New Real-Time Heartbeat Failure Detector. Wireless Communications, Networking and Mobile Computing, 2008. WiCOM '08. 4th International Conference on. 2008. Page(s): $1-3$. 